\title{
On bounds in Poisson approximation for distributions of independent negative-binomial distributed random variables
}

\author{
Tran Loc Hung * and Le Truong Giang
}

*Correspondence: tlhungvn@gmail.com; tlhung@ufm.edu.vn University of Finance and Marketing, 306 Nguyen Trong Tuyen Street, Tan Binh District, Ho Chi Minh City, Vietnam

\begin{abstract}
Using the Stein-Chen method some upper bounds in Poisson approximation for distributions of row-wise triangular arrays of independent negative-binomial distributed random variables are established in this note.
\end{abstract}

Keywords: Stein-Chen method, Poisson approximation, Le Cam's inequality, Negative-binomial variable

Mathematics Subject Classification: 60F05, 60G50, 41A36

\section{Background}

Let $X_{n, 1}, X_{n, 2}, \ldots ; n=1,2, \ldots$ be a row-wise triangular array of independent negativebinomial distributed random variables with probabilities

$$
P\left(X_{n, i}=k\right)=C_{r_{n, i}+k-1}^{k}\left(1-p_{n, i}\right)^{k} p_{n, i}^{r_{n, i}},
$$

where $p_{n, i} \in(0,1) ; r_{n, i}=1,2, \ldots ; i=1,2, \ldots ; k=0,1, \ldots$ It is worth pointing out that if all $r_{n, 1}=r_{n, 2}=\cdots=1 ; n=1,2, \ldots$, then we have the sequence of independent geometric distributed random variables with success probabilities $p_{n, 1}, p_{n, 2}, \ldots ; n=1,2, \ldots$ Write $W_{n}=\sum_{i=1}^{n} X_{n, i}$ and $\lambda_{n}=E\left(W_{n}\right)=\sum_{i=1}^{n} r_{n, i}\left(1-p_{n, i}\right) p_{n, i}^{-1}$. We will denote by $Z_{\lambda_{n}}$ the Poisson random variable with positive mean $\lambda_{n}$.

The main aim of this paper is to establish some upper bounds in Poisson approximation for $\sum_{k=1}^{\infty}\left|P\left(W_{n}=k\right)-P\left(Z_{\lambda_{n}}=k\right)\right|$ for the sequence $X_{n, 1}, X_{n, 2}, \ldots ; n=1,2, \ldots$ by the well-known Stein-Chen method.

It has long been known that the remarkable Le Cam's inequality in Poisson approximation for the row-wise triangular array of independent Bernoulli distributed random variables $Y_{n, 1}, Y_{n, 2}, \ldots ; n=1,2, \ldots$ with probabilities $P\left(Y_{n, i}=1\right)=p_{n, i}=1-P\left(Y_{n, i}=0\right), i=1,2, \ldots$ is defined as follows:

$$
\sum_{k=1}^{\infty}\left|P\left(S_{n}=k\right)-P\left(Z_{\beta_{n}}=k\right)\right| \leq 2 \sum_{i=1}^{n} p_{n, i}^{2},
$$

where $S_{n}=\sum_{i=1}^{n} Y_{n, i}$ and $\beta_{n}=E\left(S_{n}\right)=\sum_{i=1}^{n} p_{n, i}$ [see Le Cam (1960), Neammanee (2003) for more details]. Moreover, a shape inequality has been established as follows:

(c) 2016 Hung and Giang. This article is distributed under the terms of the Creative Commons Attribution 4.0 International License (http://creativecommons.org/licenses/by/4.0/), which permits unrestricted use, distribution, and reproduction in any medium, provided you give appropriate credit to the original author(s) and the source, provide a link to the Creative Commons license, and indicate if changes were made. 


$$
\sum_{k=1}^{\infty}\left|P\left(S_{n}=k\right)-P\left(Z_{\beta_{n}}=k\right)\right| \leq \frac{2\left(1-e^{-\beta_{n}}\right)}{\beta_{n}} \sum_{i=1}^{n} p_{n, i}^{2} .
$$

[We refer the reader to Barbour et al. (1992) and Chen (1975)]. As far as we know the Stein-Chen method is the well-known method have been used in Poisson approximation problems and it can be applied to a wide class of discrete random variables as geometric distributed random variables and negative-binomial distributed random variables. In recent years, using the Stein-Chen method, many results related to Poisson approximation for various discrete random variables are established in Teerapabolarn and Wongkasem (2007), Teerapabolarn $(2009,2013)$. These results are included here for the sake of completeness. Let $Z_{1}, Z_{2}, \ldots$ be a sequence of independent geometric distributed random variables with probabilities $P\left(Z_{i}=k\right)=\left(1-p_{i}\right)^{k} p_{i}, k=0,1,2, \ldots ; i=1,2, \ldots$ Then, for $A \subseteq \mathbb{Z}_{+}:=\{0,1,2, \ldots\}$,

$$
\sup _{A}\left|P\left(V_{n} \in A\right)-\sum_{k \in A} \frac{\gamma_{n}{ }^{k} e^{-\gamma_{n}}}{k !}\right| \leq \sum_{i=1}^{n} \min \left\{\frac{\gamma_{n}{ }^{-1}\left(1-e^{-\gamma_{n}}\right)}{p_{i}}, 1\right\}\left(1-p_{i}\right)^{2} p_{i}^{-1},
$$

and for $A \subseteq \mathbb{Z}_{+}, w_{0} \in \mathbb{Z}_{+}$

$\left|P\left(V_{n} \leq w_{0}\right)-\sum_{k=0}^{w_{0}} \frac{\gamma_{n}{ }^{k} e^{-\gamma_{n}}}{k !}\right| \leq \gamma_{n}{ }^{-1}\left(e^{-\gamma_{n}}-1\right) \sum_{i=1}^{n} \min \left\{\frac{1}{p_{i}\left(w_{0}+1\right)}, 1\right\}\left(1-p_{i}\right)^{2} p_{i}^{-1}$,

where $V_{n}=\sum_{i=1}^{n} Z_{i}, \gamma_{n}=E\left(V_{n}\right)=\sum_{i=1}^{n}\left(1-p_{i}\right) p_{i}^{-1}$ [see Teerapabolarn and Wongkasem (2007), for more details]. It should be noted that in case when the mean $\gamma_{n}=E\left(V_{n}\right)$ will be replaced by a parameter $\overline{\gamma_{n}}=\sum_{i=1}^{n}\left(1-p_{i}\right)$, another results will be established as follows:

$-{\overline{\gamma_{n}}}^{-1}\left(e^{-\overline{\gamma_{n}}}-1\right) \sum_{i=1}^{n} \min \left\{\frac{1}{p_{i}\left(w_{0}+1\right)}, 1\right\}\left(1-p_{i}\right)^{2} \leq P\left(V_{n} \leq w_{0}\right)-\sum_{k=0}^{w_{0}} \frac{{\overline{\gamma_{n}}}^{k} e^{-\overline{\gamma_{n}}}}{k !} \leq 0$

and

$$
\begin{aligned}
& \left|P\left(V_{n} \leq w_{0}\right)-\sum_{k=0}^{w_{0}} \frac{e^{-\overline{\gamma_{n}}}{\overline{\gamma_{n}}}^{k}}{k !}\right| \\
& \quad \leq \frac{\sum_{k=0}^{w_{0}} \frac{e^{-\overline{\gamma_{n}} \bar{\gamma}_{n}^{k}}}{k !}\left(1-\sum_{k=0}^{w_{0}} \frac{\left.e^{-\overline{\gamma_{n}}} \overline{\bar{\gamma}_{n}{ }^{k}}\right)}{k !} \sum_{i=1}^{n} \min \left\{\frac{1}{p_{i}\left(w_{0}+1\right)}, 1\right\}\left(1-p_{i}\right)^{2},\right.}{\left(w_{0}+1\right) !}
\end{aligned}
$$

for $A \subseteq \mathbb{Z}_{+}$[results of this nature may be found in Teerapabolarn (2013)]. It is easy to check that when the values $r_{n, 1}=r_{n, 2}=\cdots=1 ; n=1,2, \ldots$ the desired sequence $\left(X_{n}, n \geq 1\right)$ will become the sequence $Z_{1}, Z_{2}, \ldots$. Therefore, it makes sense to consider the results in (4), (5), (6), and (7) for negative-binomial random variables with probabilities in term of (1).

It should be noted that in recent years the same problem was tackled in Upadhye and Vellaisamy (2014) and Vellaisamy and Upadhye (2009) by using Kerstans method (1964) and the method of exponents [see Upadhye and Vellaisamy $(2013,2014)$ and Vellaisamy 
and Upadhye (2009), for more details]. The compound negative binomial and compound Poisson approximations to the generalized Poisson binomial distribution are studied and applications are also discussed [see Upadhye and Vellaisamy $(2013,2014)$, for more details]. Specifically, using Kerstans method (1964) and the method of exponents, Vellaisamy and Upadhye (2009) have established the bounds in Poisson approximation as following inequality:

$$
d_{T V}\left(S_{n}, Z_{\lambda}\right) \leq \sum_{j=1}^{n} \frac{\alpha_{j} q_{j}^{2}}{p_{j}} \min \left\{1, \frac{1}{\sqrt{2 \lambda e}}\right\}
$$

where $\lambda=\sum_{i=1}^{n} \alpha_{i} q_{i}=\alpha q$, for $X_{1}, X_{2}, \ldots, X_{n}$ are independent negative binomial distributed random variables with parameters $\alpha_{j}$ and $q_{j}, j=1,2, \ldots, n$ and $Z_{\lambda}$ is a Poisson random variable with mean $\lambda$.

It is worth pointing out that comparison of bounds in negative binomial approximation and Poisson approximation is showing that an negative binomial approximation is better than Poisson approximation in the case $X_{j}, j=1,2, \ldots$ are independent negative binomial random variables [see Theorem 2.2 and Theorem 2.4 in Vellaisamy and Upadhye (2009)].

Besides, Poisson approximation is also considered for a wide class of discrete random variables via operator method and method of probability distance [see Hung and Thao (2013) and Hung and Giang (2014), for more details].

The main purpose of this paper is to use the Stein-Chen method for providing the bounds of Le Cam-type inequality (2) and (3) in Poisson approximation for row-wise arrays of independent negative-binomial distributed random variables. The results obtained in this paper are extensions and generalizations of some results in Teerapabolarn and Wongkasem (2007), Teerapabolarn (2009, 2013).

\section{Preliminaries}

During the last several decades the Stein-Chen method has risen to become one of the most important tools available for studying in Poisson approximation problems. The Stein-Chen method has been dealt with in detail in many articles [the reader is referred to Stein (1972), Chen (1975), Chen and Röllin (2013), Barbour et al. (1992) and Barbour and Chen (2004) for fuller development]. The Stein-Chen method can be summarized as follows:

Let us denote by $F_{X}(A)$ the probability distribution function of a discrete random variable $X \in A$ and we will denoted by $P_{\alpha_{n}}(A)=\sum_{k \in A} e^{-\alpha_{n}} \frac{\alpha_{n}{ }^{k}}{k !}$ the Poisson distribution function, defined on the set $A \subseteq \mathbb{Z}_{+}$. The best known method for estimating

$$
\Delta=\sup _{x}\left|F_{X}(A)-P_{\alpha_{n}}(A)\right|
$$

is basing on the following arguments [see Chen (1975) for more details]:

Assume that $h(u)$ is a real-valued bounded function and $P_{\alpha_{n}} h=e^{-\alpha_{n}} \sum_{k=0}^{\infty} h(k) \frac{\alpha_{n}{ }^{k}}{k !}$. Consider the function $f($.) which is a solution of the differential equation

$$
\alpha_{n} f(x+1)-x f(x)=h(x)-P_{\alpha_{n}} h .
$$


Setting

$$
h(x)=h_{A}(x)= \begin{cases}1, & \text { if } x \in A \\ 0, & \text { if } x \notin A\end{cases}
$$

Putting $x=X$ and taking the expectation of both sides of the above differential equation, we have

$$
F_{X}(A)-P_{\alpha_{n}}(A)=E\left[\alpha_{n} f(X+1)-X f(X)\right] .
$$

Thus, the problem of estimating $\Delta$ can be reduced to that of estimating the difference of the expectations

$$
\left|E \alpha_{n} f(X+1)-E X f(X)\right| .
$$

Before starting the main results in the next section we first recall the following remarkable lemmas:

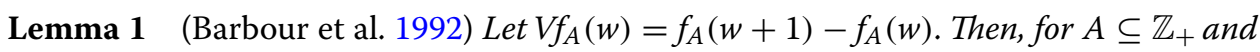
$k \in \mathbb{Z}_{+} \backslash\{0\}$,

$$
\sup _{w \geq k}\left|V f_{A}(w)\right| \leq \min \left\{\alpha_{n}^{-1}\left(1-e^{-\alpha_{n}}\right), \frac{1}{k}\right\}
$$

Lemma 2 (Teerapabolarn and Wongkasem 2007) Let $w_{0} \in \mathbb{Z}_{+}$and $k \in \mathbb{Z}_{+} \backslash\{0\}$, we have

$$
\sup _{w \geq k}\left|V f_{C_{w_{0}}}(w)\right| \leq \gamma_{n}^{-1}\left(e^{\gamma_{n}}-1\right) \min \left\{\frac{1}{w_{0}+1}, \frac{1}{k}\right\}
$$

Lemma 3 (Teerapabolarn 2009) Let $w_{0} \in \mathbb{Z}_{+}$and $k \in \mathbb{Z}_{+} \backslash\{0,1\}$. Then, we have

$$
0<\sup _{w \geq k} f(w) \leq{\overline{\gamma_{n}}}^{-1}\left(e^{\overline{\gamma_{n}}}-1\right) \min \left\{\frac{1}{k}, \frac{1}{w_{0}+1}\right\} .
$$

Lemma 4 (Teerapabolarn 2013) For $w_{0} \in \mathbb{Z}_{+}$and $k \in \mathbb{Z}_{+} \backslash\{0,1\}$, let $p_{\overline{\gamma_{n}}}\left(w_{0}\right)=\frac{e^{-\overline{\gamma_{n}}} \overline{\gamma_{n}} w_{0}}{w_{0} !}$ and $P_{\overline{\gamma_{n}}}\left(w_{0}\right)=\sum_{k=0}^{w_{0}} \frac{{\overline{\gamma_{n}}}^{k} e^{-\overline{\gamma_{n}}}}{k !}$. Then the following inequality is true

$$
\sup _{w \geq k} f_{C_{w_{0}}}(w) \leq \frac{P_{\overline{\gamma_{n}}}\left(w_{0}\right)\left(1-P_{\overline{\gamma_{n}}}\left(w_{0}\right)\right)}{p_{\overline{\gamma_{n}}}\left(w_{0}+1\right)} \min \left\{\frac{1}{w_{0}+1}, \frac{1}{k}\right\} .
$$

\section{Results}

Throughout the forthcoming, unless otherwise specified, we shall denote by $X_{n, 1}, X_{n, 2}, \ldots ; n=1,2, \ldots$ a row-wise triangular array of independent negative-binomial distributed random variables with probabilities

$$
P\left(X_{n, i}=k\right)=C_{r_{n, i}+k-1}^{k}\left(1-p_{n, i}\right)^{k} p_{n, i}^{r_{n, i}}
$$


where $p_{n, i} \in(0,1) ; r_{n, i}=1,2, \ldots ; i=1,2, \ldots ; k=0,1, \ldots$ Let $W_{n}=\sum_{i=1}^{n} X_{n, i}$ and set $\lambda_{n}=E\left(W_{n}\right)=\sum_{i=1}^{n} r_{n, i}\left(1-p_{n, i}\right) p_{n, i}^{-1}$. Then, for $r_{n, i} \in\{1,2, \ldots\}$ we have the following theorems:

Theorem 1 For $A \subseteq \mathbb{Z}_{+}$,

$$
\begin{aligned}
& \sup _{A}\left|P\left(W_{n} \in A\right)-\sum_{k \in A} \frac{\lambda_{n}^{k} e^{-\lambda_{n}}}{k !}\right| \\
& \leq \sum_{i=1}^{n} \min \left\{\lambda_{n}^{-1}\left(1-e^{-\lambda_{n}}\right) r_{n, i}\left(1-p_{n, i}\right) p_{n, i}^{-1}, 1-p_{n, i}^{r_{n, i}}\right\}\left(1-p_{n, i}\right) p_{n, i}^{-1} .
\end{aligned}
$$

Proof Let $\mathrm{f}$ and $\mathrm{h}$ are bounded real-valued functions defined on $\mathbb{Z}_{+}$. For $w=0,1, \ldots$ we have the Stein's equation for Poisson distribution with a mean $\lambda_{n}$

$$
\lambda_{n} f(w+1)-w f(w)=h(w)-P_{\lambda_{n}}(h),
$$

where $P_{\lambda_{n}}(h)=e^{-\lambda_{n}} \sum_{k=0}^{\infty} h(k) \frac{\lambda_{n}{ }^{k}}{k !}$.

For $A \subseteq \mathbb{Z}_{+}$, let us denote by $h_{A}: \mathbb{Z}_{+} \rightarrow \mathbb{R}$ and by $f_{A}(w)$ the functions defined by

$$
h_{A}(w)= \begin{cases}1, & \text { if } w \in A \\ 0, & \text { if } w \notin A\end{cases}
$$

and

$$
f_{A}(w)= \begin{cases}(w-1) ! \lambda_{n}{ }^{-w} e^{\lambda_{n}}\left[P_{\lambda_{n}}\left(h_{A \cap C_{w-1}}\right)-P_{\lambda_{n}}\left(h_{A}\right) P_{\lambda_{n}}\left(h_{C_{w-1}}\right)\right], & \text { if } w \geq 1 \\ 0, & \text { if } w=0\end{cases}
$$

where $C_{w}=\{0,1, \cdots, w\}$.

Given $f=f_{A}$ and $h=h_{A}$, We have the following Stein's equation:

$$
\lambda_{n} f(w+1)-w f(w)=h_{A}(w)-P_{\lambda_{n}}\left(h_{A}\right),
$$

where

$$
P_{\lambda_{n}}\left(h_{A}\right)=e^{-\lambda_{n}} \sum_{k=0}^{\infty} h_{A}(k) \frac{\lambda_{n}^{k}}{k !}=\sum_{k \in A} e^{-\lambda_{n}} \frac{\lambda_{n}^{k}}{k !} .
$$

Therefore, the Stein's equation can be written as follows:

$$
h_{A}(w)-\sum_{k \in A} e^{-\lambda_{n}} \frac{\lambda_{n}^{k}}{k !}=\lambda_{n} f(w+1)-w f(w) .
$$

Taking expectations of both sides of above equation, we have

$$
P\left(W_{n} \in A\right)-\sum_{k \in A} \frac{\lambda_{n}^{k} e^{-\lambda_{n}}}{k !}=E\left[\lambda_{n} f\left(W_{n}+1\right)-W_{n} f\left(W_{n}\right)\right] .
$$

It follows that 


$$
\begin{aligned}
& \left|P\left(W_{n} \in A\right)-\sum_{k \in A} \frac{\lambda_{n}^{k} e^{-\lambda_{n}}}{k !}\right|=E\left[\lambda_{n} f\left(W_{n}+1\right)-W_{n} f\left(W_{n}\right)\right] \mid \\
& \quad \leq \sum_{i=1}^{n}\left|E\left[r_{n, i}\left(p_{n, i}^{-1}-1\right) f\left(W_{n}+1\right)-X_{n, i} f\left(W_{n}\right)\right]\right| .
\end{aligned}
$$

Let $W_{i}=W_{n}-X_{n, i}$. Then, for each $i$, we get

$$
\begin{aligned}
& E\left[r_{n, i}\left(p_{n, i}^{-1}-1\right) f\left(W_{n}+1\right)-X_{n, i} f\left(W_{n}\right)\right] \\
& =E\left[r_{n, i}\left(p_{n, i}^{-1}-1\right) f\left(W_{i}+X_{n, i}+1\right)-X_{n, i} f\left(W_{i}+X_{n, i}\right)\right] \\
& =E\left[E\left[\left(r_{n, i}\left(p_{n, i}^{-1}-1\right) f\left(W_{i}+X_{n, i}+1\right)-X_{n, i} f\left(W_{i}+X_{n, i}\right)\right) \mid X_{n, i}\right]\right] \\
& =E\left[\left(r_{n, i}\left(p_{n, i}^{-1}-1\right) f\left(W_{i}+X_{n, i}+1\right)-X_{n, i} f\left(W_{i}+X_{n, i}\right)\right) \mid X_{n, i}=0\right] p_{n, i}^{r_{n, i}} \\
& +E\left[\left(r_{n, i}\left(p_{n, i}^{-1}-1\right) f\left(W_{i}+X_{n, i}+1\right)-X_{n, i} f\left(W_{i}+X_{n, i}\right)\right) \mid X_{n, i}=1\right] r_{n, i} p_{n, i}^{r_{n, i}}\left(1-p_{n, i}\right) \\
& +\sum_{k \geq 2} E\left[\left(r_{n, i}\left(p_{n, i}^{-1}-1\right) f\left(W_{i}+X_{n, i}+1\right)\right.\right. \\
& \left.\left.-X_{n, i} f\left(W_{i}+X_{n, i}\right)\right) \mid X_{n, i}=k\right] C_{r_{n, i}+k-1}^{k} p_{n, i}^{r_{n, i}}\left(1-p_{n, i}\right)^{k} \\
& =E\left[r_{n, i}\left(p_{n, i}^{-1}-1\right) p_{n, i}^{r_{n, i}} f\left(W_{i}+1\right)\right] \\
& +E\left[r_{n, i}^{2}\left(1-p_{n, i}\right)^{2} p_{n, i}^{r_{n, i}-1} f\left(W_{i}+2\right)-r_{n, i} p_{n, i}^{r_{n, i}}\left(1-p_{n, i}\right) f\left(W_{i}+1\right)\right] \\
& +\sum_{k \geq 2} E\left[C_{r_{n, i}+k-1}^{k} r_{n, i}\left(1-p_{n, i}\right)^{k+1} p_{n, i}^{r_{n, i}-1} f\left(W_{i}+k+1\right)\right. \\
& \left.-k C_{r_{n, i}+k-1}^{k} p_{n, i}^{r_{n, i}}\left(1-p_{n, i}\right)^{k} f\left(W_{i}+k\right)\right] \\
& =r_{n, i}\left(1-p_{n, i}\right)^{2} p_{n, i}^{r_{n, i}-1} E\left[f\left(W_{i}+1\right)\right]+E\left[r_{n, i}^{2}\left(1-p_{n, i}\right)^{2} p_{n, i}^{r_{n, i}-1} f\left(W_{i}+2\right)\right] \\
& +\sum_{k \geq 2} E\left[C_{r_{n, i}+k-1}^{k} r_{n, i}\left(1-p_{n, i}\right)^{k+1} p_{n, i}^{r_{n, i}-1} f\left(W_{i}+k+1\right)\right. \\
& \left.-k C_{r_{n, i}+k-1}^{k} p_{n, i}^{r_{n, i}}\left(1-p_{n, i}\right)^{k} f\left(W_{i}+k\right)\right] \\
& =r_{n, i}\left(1-p_{n, i}\right)^{2} p_{n, i}^{r_{n, i}-1} E\left[f\left(W_{i}+1\right)\right] \\
& +\sum_{k \geq 2} E\left[C_{r_{n, i}+k-2}^{k-1} r_{n, i}\left(1-p_{n, i}\right)^{k} p_{n, i}^{r_{n, i}-1} f\left(W_{i}+k\right)-k C_{r_{n, i}+k-1}^{k} p_{n, i}^{r_{n, i}}\left(1-p_{n, i}\right)^{k} f\left(W_{i}+k\right)\right] \\
& =r_{n, i}\left(1-p_{n, i}\right)^{2} p_{n, i}^{r_{n, i}-1} E\left[f\left(W_{i}+1\right)\right] \\
& +\sum_{k \geq 2} E\left[C_{r_{n, i}+k-2}^{k-1} r_{n, i}\left(1-p_{n, i}\right)^{k} p_{n, i}^{r_{n, i}-1} f\left(W_{i}+k\right)\right. \\
& \left.-\left(r_{n, i}+k-1\right) C_{r_{n, i}+k-2}^{k-1} p_{n, i}^{r_{n, i}}\left(1-p_{n, i}\right)^{k} f\left(W_{i}+k\right)\right]
\end{aligned}
$$




$$
\begin{aligned}
& =r_{n, i}\left(1-p_{n, i}\right)^{2} p_{n, i}^{r_{n, i}-1} E\left[f\left(W_{i}+1\right)\right] \\
& +\sum_{k \geq 2} E\left[\frac{r_{n, i}+k-1}{r_{n, i}} C_{r_{n, i}+k-2}^{k-1} r_{n, i}\left(1-p_{n, i}\right)^{k} p_{n, i}^{r_{n, i}-1} f\left(W_{i}+k\right)\right. \\
& \left.-\left(r_{n, i}+k-1\right) C_{r_{n, i}+k-2}^{k-1} p_{i}^{r_{n, i}}\left(1-p_{n, i}\right)^{k} f\left(W_{i}+k\right)\right] \\
& -\sum_{k \geq 2}\left(\frac{r_{n, i}+k-1}{r_{n, i}}-1\right) C_{r_{n, i}+k-2}^{k-1} r_{n, i}\left(1-p_{n, i}\right)^{k} p_{n, i}^{r_{n, i}-1} E\left[f\left(W_{i}+k\right)\right] \\
& =r_{n, i}\left(1-p_{n, i}\right)^{2} p_{n, i}^{r_{n, i}-1} E\left[f\left(W_{i}+1\right)\right] \\
& +\sum_{k \geq 2}\left(r_{n, i}+k-1\right) C_{r_{n, i}+k-2}^{k-1}\left(1-p_{n, i}\right)^{k+1} p_{n, i}^{r_{n, i}-1} E\left[f\left(W_{i}+k\right)\right] \\
& -\sum_{k \geq 2}\left(\frac{r_{n, i}+k}{r_{n, i}}-1\right) C_{r_{n, i}+k-1}^{k} r_{n, i}\left(1-p_{n, i}\right)^{k+1} p_{n, i}^{r_{n, i}-1} E\left[f\left(W_{i}+k+1\right)\right] \\
& -r_{n, i}\left(1-p_{n, i}\right)^{2} p_{n, i}^{r_{n, i}-1} E\left[f\left(W_{i}+2\right)\right] \\
& =r_{n, i}\left(1-p_{n, i}\right)^{2} p_{n, i}^{r_{n, i}-1} E\left[f\left(W_{i}+1\right)\right]-r_{n, i}\left(1-p_{n, i}\right)^{2} p_{n, i}^{r_{n, i}-1} E\left[f\left(W_{i}+2\right)\right] \\
& +\sum_{k \geq 2} k C_{r_{n, i}+k-1}^{k}\left(1-p_{n, i}\right)^{k+1} p_{n, i}^{r_{n, i}-1} E\left[f\left(W_{i}+k\right)\right] \\
& -\sum_{k \geq 2} k C_{r_{n, i}+k-1}^{k}\left(1-p_{n, i}\right)^{k+1} p_{n, i}^{r_{n, i}-1} E\left[f\left(W_{i}+k+1\right)\right] \\
& =r_{n, i}\left(1-p_{n, i}\right)^{2} p_{n, i}^{r_{n, i}-1} E\left[f\left(W_{i}+1\right)-f\left(W_{i}+2\right)\right] \\
& +\sum_{k \geq 2} k C_{r_{n, i}+k-1}^{k}\left(1-p_{n, i}\right)^{k+1} p_{n, i}^{r_{n, i}-1} E\left[f\left(W_{i}+k\right)-f\left(W_{i}+k+1\right)\right] \\
& =\sum_{k \geq 1} k C_{r_{n, i}+k-1}^{k}\left(1-p_{n, i}\right)^{k+1} p_{n, i}^{r_{n, i}-1} E\left[f\left(W_{i}+k\right)-f\left(W_{i}+k+1\right)\right] .
\end{aligned}
$$

By using Lemma 1, we have

$$
\begin{aligned}
\mid E & {\left[r_{n, i}\left(p_{n, i}^{-1}-1\right) f\left(W_{n}+1\right)-X_{n, i} f\left(W_{n}\right)\right] \mid } \\
\leq & \sum_{k \geq 1} k C_{r_{n, i}+k-1}^{k}\left(1-p_{n, i}\right)^{k+1} p_{n, i}^{r_{n, i}-1} E\left|f\left(W_{i}+k\right)-f\left(W_{i}+k+1\right)\right| \\
\leq & \sum_{k \geq 1} k C_{r_{n, i}+k-1}^{k}\left(1-p_{n, i}\right)^{k+1} p_{n, i}^{r_{n, i}-1} \sup _{w \geq k}|V f(w)| \\
\leq & \min \left\{\lambda_{n}{ }^{-1}\left(1-e^{-\lambda_{n}}\right) p_{n, i}^{r_{n, i}-1} \sum_{k \geq 1} k C_{r_{n, i}+k-1}^{k}\left(1-p_{n, i}\right)^{k+1},\right. \\
& \left.p_{n, i}^{r_{n, i}-1} \sum_{k \geq 1} C_{r_{n, i}+k-1}^{k}\left(1-p_{n, i}\right)^{k+1}\right\} \\
= & \min \left\{\lambda_{n}{ }^{-1}\left(1-e^{-\lambda_{n}}\right) p_{n, i}^{r_{n, i}-1}\left(1-p_{n, i}\right) \sum_{k \geq 1} k C_{r_{n, i}}^{k}+k-1\right. \\
& \left.p_{n, i}^{r_{n, i}-1}\left(1-p_{n, i}\right)\left(p_{n, i}^{-r_{n, i}}-1\right)\right\} \\
= & \min \left\{\lambda_{n}^{-1}\left(1-e^{-\lambda_{n}}\right) p_{n, i}^{r_{n, i}-1}\left(1-p_{n, i}\right) r_{n, i},\left(1-p_{n, i}\right) p_{n, i}^{-r_{n, i}-1}, p_{n, i}^{-1}\left(1-p_{n, i}\right)\left(1-p_{n, i}^{r_{n, i}}\right)\right\} \\
= & \min \left\{\lambda_{n}^{-1}\left(1-e^{-\lambda_{n}}\right) r_{n, i}\left(1-p_{n, i}\right) p_{n, i}^{-1}, 1-p_{n, i}^{r_{n, i}}\right\}\left(1-p_{n, i}\right) p_{n, i}^{-1} .
\end{aligned}
$$


To combine (8) and (9), we have

$$
\begin{aligned}
& \sup _{A}\left|P\left(W_{n} \in A\right)-\sum_{k \in A} \frac{\lambda_{n}^{k} e^{-\lambda_{n}}}{k !}\right| \\
& \quad \leq \sum_{i=1}^{n} \min \left\{\lambda_{n}^{-1}\left(1-e^{-\lambda_{n}}\right) r_{n, i}\left(1-p_{n, i}\right) p_{n, i}^{-1}, 1-p_{n, i}^{r_{n, i}}\right\}\left(1-p_{n, i}\right) p_{n, i}^{-1} .
\end{aligned}
$$

The proof is complete.

Remark 1 It is easily seen that the (4) is a special case of the Theorem 1 with $r_{n, i}=1 ; n=1,2, \ldots ; i=1,2, \ldots n$

Theorem 2 Let $W_{n}$ and $\lambda_{n}$ be defined as in Theorem 1 . Then, for $w_{0} \in \mathbb{N}$,

$$
\begin{aligned}
& \left|P\left(W_{n} \leq w_{0}\right)-\sum_{k \leq w_{0}} \frac{\lambda_{n}^{k} e^{-\lambda_{n}}}{k !}\right| \\
& \quad \leq \lambda_{n}^{-1}\left(e^{\lambda_{n}}-1\right) \sum_{i=1}^{n} \min \left\{\frac{r_{n, i}\left(1-p_{n, i}\right)}{p_{n, i}\left(w_{0}+1\right)}, 1-p_{n, i}^{r_{n, i}}\right\}\left(1-p_{n, i}\right) p_{n, i}^{-1} .
\end{aligned}
$$

Proof For $C_{w}=\{0, \ldots, w\}$ and $w_{0} \in N$, let $h_{w_{0}}: \mathbb{Z}_{+} \rightarrow \mathbb{R}, f_{C_{w_{0}}}\left(w_{0}\right)$ be defined by

$$
\begin{aligned}
& h_{C_{w_{0}}}(w)= \begin{cases}1 & \text { if } w \leq w_{0}, \\
0 & \text { if } w>w_{0} .\end{cases} \\
& f_{C_{w_{0}}}(w)= \begin{cases}(w-1) ! \lambda_{n}{ }^{-w} e^{\lambda_{n}}\left[P_{\lambda_{n}}\left(h_{C_{w 0}}\right) P_{\lambda_{n}}\left(1-h_{C_{w-1}}\right)\right] & \text { if } w_{0}<w, \\
(w-1) ! \lambda_{n}{ }^{-w} e^{\lambda_{n}}\left[P_{\lambda_{n}}\left(h_{C_{w-1}}\right) P_{\lambda_{n}}\left(1-h_{C_{w_{0}}}\right)\right] & \text { if } w_{0} \geq w, \\
0 & \text { if } w=0 .\end{cases}
\end{aligned}
$$

Given $f=f_{C_{w_{0}}}$ and $h=h_{C_{w_{0}}}$. We have the Stein's equation

$$
h_{C_{w_{0}}}(w)-\sum_{k \leq w_{0}} e^{-\lambda_{n}} \frac{\lambda_{n}^{k}}{k !}=\lambda_{n} f(w+1)-w f(w) .
$$

Taking expectations of both sides and arguing similarly to the proof of Theorem 1 we prove that

$$
\left|P\left(W_{n} \leq w_{0}\right)-\sum_{k \leq w_{0}} \frac{\lambda_{n}^{k} e^{-\lambda_{n}}}{k !}\right| \leq \sum_{i=1}^{n}\left|E\left[r_{n, i}\left(p_{n, i}^{-1}-1\right) f\left(W_{n}+1\right)-X_{n, i} f\left(W_{n}\right)\right]\right| .
$$

According to the Theorem 1, we have

$$
\begin{aligned}
& E\left[r_{n, i}\left(p_{n, i}^{-1}-1\right) f\left(W_{n}+1\right)-X_{n, i} f\left(W_{n}\right)\right] \\
& \quad=\sum_{k \geq 1} k C_{r_{n, i}+k-1}^{k}\left(1-p_{n, i}\right)^{k+1} p_{n, i}^{r_{n, i}-1} E\left[f\left(W_{i}+k\right)-f\left(W_{i}+k+1\right)\right] .
\end{aligned}
$$


Hence, by (10), (11) and Lemma 2, we have

$$
\begin{aligned}
& \left|P\left(W_{n} \leq w_{0}\right)-\sum_{k \leq w_{0}} \frac{\lambda_{n}^{k} e^{-\lambda_{n}}}{k !}\right| \leq \sum_{i=1}^{n}\left|E\left[r_{n, i}\left(p_{n, i}^{-1}-1\right) f\left(W_{n}+1\right)-X_{n, i} f\left(W_{n}\right)\right]\right| \\
& \leq \sum_{i=1}^{n}\left(\sum_{k \geq 1} k C_{r_{n, i}+k-1}^{k}\left(1-p_{n, i}\right)^{k+1} p_{n, i}^{r_{n, i}-1} \sup _{w \geq k}|V f(w)|\right) \\
& \leq \sum_{i=1}^{n}\left(\lambda _ { n } ^ { - 1 } ( e ^ { \lambda _ { n } } - 1 ) \operatorname { m i n } \left\{\frac{p_{n, i}^{r_{n, i}-1}}{w_{0}+1} \sum_{k \geq 1} k C_{r_{n, i}+k-1}^{k}\left(1-p_{n, i}\right)^{k+1},\right.\right. \\
& \left.\left.p_{n, i}^{r_{n, i}-1} \sum_{k \geq 1} C_{r_{n, i}+k-1}^{k}\left(1-p_{n, i}\right)^{k+1}\right\}\right) \\
& \leq \sum_{i=1}^{n}\left(\lambda _ { n } { } ^ { - 1 } ( e ^ { \lambda _ { n } } - 1 ) \operatorname { m i n } \left\{\frac{p_{n, i}^{r_{n, i}-1}\left(1-p_{n, i}\right)}{w_{0}+1} \sum_{k \geq 1} k C_{r_{n, i}+k-1}^{k}\left(1-p_{n, i}\right)^{k},\right.\right. \\
& \left.\left.p_{n, i}^{r_{n, i}-1}\left(1-p_{n, i}\right)\left(p_{n, i}^{-r_{n, i}}-1\right)\right\}\right) \\
& =\sum_{i=1}^{n}\left(\lambda_{n}^{-1}\left(e^{\lambda_{n}}-1\right) \min \left\{\frac{p_{n, i}^{r_{n, i}-1}\left(1-p_{n, i}\right) r_{n, i}\left(1-p_{n, i}\right)}{\left(w_{0}+1\right) p_{n, i}^{r_{n, i}+1}},\left(1-p_{n, i}\right) p_{n, i}^{-1}\left(1-p_{n, i}^{r_{n, i}}\right)\right\}\right) \\
& =\sum_{i=1}^{n}\left(\lambda_{n}^{-1}\left(e^{\lambda_{n}}-1\right) \min \left\{\frac{r_{n, i}\left(1-p_{n, i}\right)}{p_{n, i}\left(w_{0}+1\right)}, 1-p_{n, i}^{r_{n, i}}\right\}\left(1-p_{n, i}\right) p_{n, i}^{-1}\right) .
\end{aligned}
$$

Thus

$$
\begin{aligned}
& \left|P\left(W_{n} \leq w_{0}\right)-\sum_{k \leq w_{0}} \frac{\lambda_{n}^{k} e^{-\lambda_{n}}}{k !}\right| \\
& \quad \leq \lambda_{n}^{-1}\left(e^{\lambda_{n}}-1\right) \sum_{i=1}^{n} \min \left\{\frac{r_{n, i}\left(1-p_{n, i}\right)}{p_{n, i}\left(w_{0}+1\right)}, 1-p_{n, i}^{r_{n, i}}\right\}\left(1-p_{n, i}\right) p_{n, i}^{-1} .
\end{aligned}
$$

This finishes the proof.

Remark 2 It is easy to check that the (5) is a special case of Theorem 2 with $r_{n, i}=1 ; n=1,2, \ldots ; i=1,2, \ldots n$.

Theorem 3 Let $W_{n}=\sum_{i=1}^{n} X_{i}$ and $\bar{\lambda}_{n}=\sum_{i=1}^{n} r_{n, i} q_{n, i}$ with $q_{n, i}=1-p_{n, i}$. Then, we have

$$
-\bar{\lambda}_{n}^{-1}\left(e^{\overline{\lambda_{n}}}-1\right) \sum_{i=1}^{n} \min \left\{\alpha_{i}, \frac{\beta_{i}-\alpha_{i}}{w_{0}+1}\right\} \leq P\left(W_{n} \leq w_{0}\right)-\sum_{k=0}^{w_{0}} \frac{\bar{\lambda}_{n}^{k} e^{-\overline{\lambda_{n}}}}{k !} \leq 0,
$$

With $\alpha_{i}=1-p_{n, i}^{r_{n, i}}-r_{n, i} q_{n, i} p_{n, i}^{r_{n, i}}, \beta_{i}=r_{n, i}\left(p_{n, i}^{-r_{n, i}}-1-r_{n, i} q_{n, i} p_{n, i}^{r_{n, i}}\right)$.

Proof Arguing as in theorem (3), we have the Stein's equation

$$
h_{w_{0}}(w)-\sum_{k=0}^{w_{0}} e^{-\bar{\lambda}_{n}} \frac{\bar{\lambda}_{n}^{k}}{k !}=\bar{\lambda}_{n} f(w+1)-w f(w) .
$$


Taking expectations of both sides, we get

$$
\begin{aligned}
P( & \left.W_{n} \leq w_{0}\right)-\sum_{k=0}^{w_{0}} \frac{\bar{\lambda}_{n}^{k} e^{-\overline{\lambda_{n}}}}{k !} \\
& =E\left[\overline{\lambda_{n}} f\left(W_{n}+1\right)-W_{n} f\left(W_{n}\right)\right] \\
& =\sum_{i=1}^{n} E\left[r_{n, i} q_{n, i} f\left(W_{n}+1\right)-X_{n, i} f\left(W_{n}\right)\right] .
\end{aligned}
$$

Let $W_{i}=W_{n}-X_{n, i}$. Then, for each $i$, we deduce

$$
\begin{aligned}
E\left[r_{n, i} q_{n, i} f\left(W_{n}+1\right)-X_{n, i} f\left(W_{n}\right)\right] & \\
= & E\left[E\left[\left(r_{n, i} q_{n, i} f\left(W_{i}+X_{n, i}+1\right)-X_{n, i} f\left(W_{i}+X_{n, i}\right)\right) \mid X_{n, i}\right]\right] \\
= & E\left[r_{n, i} q_{n, i} p_{n, i}^{r_{n, i}} f\left(W_{i}+1\right)\right]+E\left[r_{n, i}^{2} q_{n, i}^{2} p_{n, i}^{r_{n, i}} f\left(W_{i}+2\right)-r_{n, i} q_{n, i} p_{n, i}^{r_{n, i}} f\left(W_{i}+X_{n, i}\right)\right] \\
& +\sum_{k \geq 2} E\left[r_{n, i} C_{r_{n, i}+k-1}^{k} q_{n, i}^{k+1} p_{n, i}^{r_{n, i}} f\left(W_{i}+k+1\right)-k C_{r_{n, i}+k-1}^{k} q_{n, i}^{k} p_{n, i}^{r_{n, i}} f\left(W_{i}+k\right)\right] \\
= & \sum_{k \geq 2} E\left[r_{n, i} C_{r_{n, i}+k-2}^{k-1} q_{n, i}^{k} p_{n, i}^{r_{n, i}} f\left(W_{i}+k\right)-k C_{r_{n, i}+k-1}^{k} q_{n, i}^{k} p_{n, i}^{r_{n, i}} f\left(W_{i}+k\right)\right] \\
= & \sum_{k \geq 2} E\left[\frac{r_{n, i} k}{r_{n, i}+k-1} C_{r_{n, i}+k-1}^{k} q_{n, i}^{k} p_{n, i}^{r_{n, i}} f\left(W_{i}+k\right)-k C_{r_{n, i}+k-1}^{k} q_{n, i}^{k} p_{n, i}^{r_{n, i}} f\left(W_{i}+k\right)\right] \\
= & \sum_{k} \frac{k(1-k)}{r_{n, i}+k-1} C_{r_{n, i}+k-1}^{k} q_{n, i}^{k} p_{n, i}^{r_{n, i}} f\left(W_{i}+k\right) \\
\geq & -\sum_{k} \frac{k(k-1)}{r_{n, i}+k-1} C_{r_{n, i}+k-1}^{k} q_{n, i}^{k} p_{n, i}^{r_{n, i}} \sup _{w \geq k} f(w) .
\end{aligned}
$$

By using Lemma 3, then we have

$$
\begin{aligned}
& -\sum_{k} \frac{k(k-1)}{r_{n, i}+k-1} C_{r_{n, i}+k-1}^{k} q_{n, i}^{k} p_{n, i}^{r_{n, i}} \sup _{w \geq k} f(w) \\
& \geq-{\overline{\lambda_{n}}}^{-1}\left(e^{\overline{\lambda_{n}}}-1\right) p_{n, i}^{r_{n, i}} \min \left\{\sum_{k} \frac{k-1}{r_{n, i}+k-1} C_{r_{n, i}+k-1}^{k} q_{n, i}^{k},\right. \\
& \left.\quad \frac{1}{w_{0}+1} \sum_{k} \frac{k(k-1)}{r_{n, i}+k-1} C_{r_{n, i}+k-1}^{k} q_{n, i}^{k}\right\} .
\end{aligned}
$$

Moreover, we have

$$
p_{n, i}^{r_{n, i}} \sum_{k} \frac{k-1}{r_{n, i}+k-1} C_{r_{n, i}+k-1}^{k} q_{n, i}^{k} \leq 1-p_{n, i}^{r_{n, i}}-r_{n, i} q_{n, i} p_{n, i}^{r_{n, i}}
$$

and

$$
\begin{aligned}
& p_{n, i}^{r_{n, i}} \sum_{k} \frac{k(k-1)}{r_{n, i}+k-1} C_{r_{n, i}+k-1}^{k} q_{n, i}^{k} \\
& \quad \leq r_{n, i}\left(p_{n, i}^{-r_{n, i}}-1-r_{n, i} q_{n, i} p_{n, i}^{r_{n, i}}\right)-\left(1-p_{n, i}^{r_{n, i}}-r_{n, i} q_{n, i} p_{n, i}^{r_{n, i}}\right) .
\end{aligned}
$$


Hence, by (12), (13), (14) and (15), we can assert that

$$
-\bar{\lambda}_{n}^{-1}\left(e^{\bar{\lambda}_{n}}-1\right) \sum_{i=1}^{n} \min \left\{\alpha_{i}, \frac{\beta_{i}-\alpha_{i}}{w_{0}+1}\right\} \leq P\left(W_{n} \leq w_{0}\right)-\sum_{k=0}^{w_{0}} \frac{\bar{\lambda}_{n}^{k} e^{-\overline{\lambda_{n}}}}{k !} \leq 0 .
$$

The proof is complete.

Remark 3 When $r_{n, i}=1$, we have

$\alpha_{i}=1-p_{n, i}-q_{n, i} p_{n, i}=\left(1-p_{n, i}\right)\left(1-p_{n, i}\right)=q_{n, i}^{2}$,

$\beta_{i}=p_{n, i}^{-1}-1-q_{n, i} p_{n, i}=\frac{1-p_{n, i}}{p_{n, i}}-\left(1-p_{n, i}\right) p_{n, i}=\frac{\left(1-p_{n, i}\right)\left(1-p_{n, i}^{2}\right)}{p_{n, i}}=q_{n, i}^{2} \frac{1+p_{n, i}}{p_{n, i}}$

$\beta_{i}-\alpha_{i}=q_{n, i}^{2}\left(\frac{1+p_{n, i}}{p_{n, i}}-1\right)=\frac{q_{n, i}^{2}}{p_{n, i}}$

It is clear that the (6) is a special case of Theorem 3 with $r_{n, i}=1 ; n=1,2, \ldots ; i=1,2, \ldots n$.

Theorem 4 Let $W_{n}=\sum_{i=1}^{n} X_{n, i}$ and $\overline{\lambda_{n}}=\sum_{i=1}^{n} r_{n, i} q_{i}$ with $q_{n, i}=1-p_{n, i}$. Then, for $w_{0} \in \mathbb{N}$ we have

$$
\left|P\left(W_{n} \leq w_{0}\right)-P_{\overline{\lambda_{n}}}\left(w_{0}\right)\right| \leq \frac{P_{\lambda_{n}}\left(w_{0}\right)\left(1-P_{\overline{\lambda_{n}}}\left(w_{0}\right)\right)}{p_{\overline{\lambda_{n}}}\left(w_{0}+1\right)} \sum_{i=1}^{n} \min \left\{\alpha_{i}, \frac{\beta_{i}-\alpha_{i}}{w_{0}+1}\right\}
$$

where

$$
\alpha_{i}=1-p_{n, i}^{r_{n, i}}-r_{n, i} q_{n, i} p_{n, i}^{r_{n, i}} \text { and } \beta_{i}=r_{n, i}\left(p_{n, i}^{-r_{n, i}}-1-r_{n, i} q_{n, i} p_{n, i}^{r_{n, i}}\right)
$$

Proof According to Theorem 3 we obtain the following inequality

$$
\left|P\left(W_{n} \leq w_{0}\right)-P_{\overline{\lambda_{n}}}\left(w_{0}\right)\right| \leq \sum_{i=1}^{n} \sum_{k} \frac{k(k-1)}{r_{n, i}+k-1} C_{r_{n, i}+k-1}^{k} q_{n, i}^{k} p_{n, i}^{r_{n, i}} \sup _{w \geq k} f_{C_{w_{0}}}(w) .
$$

By using Lemma 4, then we have

$$
\begin{aligned}
& \left|P\left(W_{n} \leq w_{0}\right)-P_{\overline{\lambda_{n}}}\left(w_{0}\right)\right| \\
& \quad \leq \frac{P_{\overline{\lambda_{n}}}\left(w_{0}\right)\left(1-P_{\overline{\lambda_{n}}}\left(w_{0}\right)\right)}{p_{\overline{\lambda_{n}}}\left(w_{0}+1\right)} \sum_{i=1}^{n} \sum_{k} \frac{k(k-1)}{r_{n, i}+k-1} C_{r_{n, i}+k-1}^{k} q_{n, i}^{k} p_{n, i}^{r_{n, i}} \min \left\{\frac{1}{w_{0}+1}, \frac{1}{k}\right\} \\
& \quad \leq \frac{P_{\overline{\lambda_{n}}}\left(w_{0}\right)\left(1-P_{\overline{\lambda_{n}}}\left(w_{0}\right)\right)}{p_{\overline{\lambda_{n}}}\left(w_{0}+1\right)} \sum_{i=1}^{n} \min \left\{\alpha_{i}, \frac{\beta_{i}-\alpha_{i}}{w_{0}+1}\right\}
\end{aligned}
$$

with $\alpha_{i}=1-p_{n, i}^{r_{n, i}}-r_{n, i} q_{n, i} p_{n, i}^{r_{n, i}}, \beta_{i}=r_{n, i}\left(p_{n, i}^{-r_{n, i}}-1-r_{n, i} q_{n, i} p_{n, i}^{r_{n, i}}\right)$

Hence, the theorem is proved. 
Remark 4 In the same way as in Remarks 3, we notice that (7) is a special case of Theorem 4 with $r_{n, i}=1 ; n=1,2, \ldots ; i=1,2, \ldots n$.

\section{Conclusions}

We conclude this paper with the following comments. The received results in this paper are extensions and generalizations of results in Teerapabolarn and Wongkasem (2007), Teerapabolarn $(2009,2013)$. The results would be more interesting and valuable if the discussed negative-binomial random variables in this paper are dependent. We shall take this up in the next study.

Authors' contributions

All authors contributed equally and significantly to this work. All authors drafted the manuscript. Both authors read and approved the final manuscript.

\section{Acknowledgements}

The authors would like to express their gratitude to the referees for valuable comments and suggestions. The research was supported by the Vietnam's National Foundation for Science and Technology Development (NAFOSTED, Vietnam) under Grant 101.01-2010.02.

\section{Competing interests}

The authors declare that they have no competing interests.

Received: 22 June 2015 Accepted: 12 January 2016

Published online: 26 January 2016

\section{References}

Barbour AD, Holst L, Janson S (1992) Poisson approximation. Clarendon Press, Oxford

Barbour AD, Chen LHY (2004) An introduction to Stein's method, Lecture Notes Series, Institute for Mathematical Sciences. National University of Singapore, vol 4

Chen LHY (1975) Poisson approximation for dependent trials. Ann Probab 3:534-545

Chen LHY, Röllin A (2013) Approximating dependent rare events. Bernoulli 19(4):1243-1267

Hung TL, Thao VT (2013) Bounds for the Approximation of Poisson-binomial distribution by Poisson distribution. J Inequal Appl 2013:30

Hung TL, Giang LT (2014) On bounds in Poisson approximation for integer-valued independent random variables. J Inequal Appl 2014:291

Kerstan J (1964) Verallgemeinerung eines Satzes von Prochorow und Le Cam. Z Wahrsch Verw Gebiete 2:173-179

Le Cam L (1960) An approximation theorem for the Poisson binomial distribution. Pacific J Math 10:1181-1197

Neammanee K (2003) A nonuniform bound for the approximation of Poisson binomial by Poisson distribution. IJMMS 48:3041-3046

Stein CM (1972) A bound for the error in normal approximation to the distribution of a sum of dependent random variables. In: Proceedings of sixth Berkeley symposium mathematical statistics and probability, vol 3, pp 583-602

Teerapabolarn K, Wongkasem P (2007) Poisson approximation for independent geometric random variables. Int Math Forum 2:3211-3218

Teerapabolarn K (2009) A note on Poisson approximation for independent geometric random variables. Int Math Forum 4:531-535

Teerapabolarn K (2013) A new bound on Poisson approximation for independent geometric variables. Int J Pure Appl Math 84(4):419-422

Upadhye NS, Vellaisamy P (2013) Improved bounds for approximations to compound distributions. Stat Probab Lett 83(2):467-473

Upadhye NS, Vellaisamy P (2014) Compound Poisson approximation to convolutions of compound negative binomial variables. Methodol Comput Appl Probab 16(4):951-968

Vellaisamy P, Upadhye NS (2009) Compound negative binomial approximations for sums of random variables. Probab Math Stat 29(2):205-226 